\title{
The Galactic Black Holes Cyg X-1, GX 339-4, and 4U 1957+11: In Transition and at High Resolution
}

\section{Michael A. Nowak*}

Massachusetts Institute of Technology, Kavli Institute for Astrophysics and Space Research E-mail: mnowakespace.mit.edu

I review here some of the open questions regarding the geometry and emission mechanisms of galactic black hole candidates. For hard states, I concentrate on the perspective of "disk+Compton coronae" models (for discussions of jet models, see the papers by Sera Markoff). Specifically, I discuss the implications from our 10 year long RXTE monitoring campaign of Cyg X-1. I then present simultaneous $R X T E / C h a n d r a$ observations of the "soft state" black hole candidate $4 \mathrm{U} 1957+11$, and discuss to what extent it does or does not allow one to test "relativistic disk models". The use of such models has been claimed to measure black hole spin parameters. I then briefly present a particularly freaky-weird observation of GX339-4, where the source "fell off" the usual radio/X-ray correlation in the low/hard state. Questions addressed by the above observations include: are the Compton corona models unique fits to the data? (No. Jets work equally well, and simple broken power laws work better still. We argue that the latter models indicate multiple, broad-band continuum components.) Is there good evidence for a receding disk as sources transit into the hard state? (The jury is still out.) What does the relativistically broadened Fe line tell us? (Sometimes the disk, even into quiescence, stays very close to the central object, in contrast to expectations of ADAF models.) How much better/more necessary are recently discussed relativistic disk models? (I am very doubtful that such models will ever usefully measure black hole spin.)

VI Microquasar Workshop: Microquasars and Beyond

September 18-22 2006

Società del Casino, Como, Italy

\footnotetext{
* Speaker.
} 


\section{Introduction}

As of this, the sixth conference in this series, I believe that most of us are comfortable with the notion that all black hole candidates (BHC) are, have been, or potentially could be microquasars, i.e., galactic compact object sources that show significant jet activity (usually in the radio). The main question that determines whether or not BHC exhibit steady jet activity is whether or not they are in the spectrally hard (i.e., "low") state [8, 3]. (For an unusual quasi-exception to this, the reader is referred to the observation of GX339-4 discussed at the end of this article.) The questions many of us are now focused on are: What are the emission mechanisms responsible for BHC hard states (corona and/or jet)? What is the hard state geometry? (The latter is especially relevant to coronal models of hard states.) Is rapid black hole spin required for launching a jet? (As the Fender Conjecture states: if jets require rapid spin, then all black holes are rapidly spinning, since all seem to exhibit jets in their hard state.) Can we measure black hole spin?

In the following, I address some of these issues via a series of pointed monitoring observations of three BHC: Cyg X-1, 4U 1957+11, and GX339-4. We have been monitoring Cyg X-1 with RXTE for nearly 10 years now, and what follows are some highlights from our work which can be read in detail elsewhere [6, 18, 24, 10, 9, 31] that are particularly relevant to Comptonization and jet models. Next, I discuss recent Chandra and RXTE observations of the soft state BHC, 4U 1957+11 (Nowak, et al., in prep.). As this source is persistently soft, with minimal hard tail emission [20, 29], it becomes an excellent test of notions of spectrally measuring spin (despite, as we shall discuss, lack of knowledge of its distance or mass). Finally, I will briefly show an unusual, radio under-luminous, hard state of GX339-4 (Nowak et al., in prep.).

Throughout, with one exception, all plotted spectra are shown as "flux-corrected" spectra (i.e., adjusted solely based upon the detector arf and $\mathrm{rmf}$, with no reference to the model), as calculated with the $I S I S^{1}$ analysis system [13]. As such, and in contrast to XSPEC "unfolded" spectra, you will not see assumed model features falsely mirrored in the data. The data are what they are, and any observed structure in the "flux-corrected" spectra are indicative of physical reality and/or response matrix features (not all of which are necessarily properly characterized).

\section{Cyg X-1}

Cyg X-1 is perhaps the most famous and one of the best studied of the BHC. A review of the overall system properties can be found in [18]. Cyg X-1 served as one of the original motivators for the definition of the "hard" (i.e., "low") and "soft" (i.e., "high") states, despite two tremendous drawbacks. First, being at least partially wind-fed rather than strictly Roche lobe-fed (i.e., "focused-wind accretion"), it likely has something of a truncated accretion disk, different than the typical X-ray nova accretion disk. Second, Cyg X-1 is perhaps one of the worst examples of "soft state" transitions. As we will discuss, the Cyg X-1 state transitions are more of degree than kind (see Fig. 2), with Cyg X-1 never entering a "disk dominated" state.

\footnotetext{
${ }^{1} A l l$ the models of XSPEC, with most of the programmability of IDL or MATLAB, plus arbitrarily and easily extendible with almost any Fortran, $C$, or $C++$ library, plus parallel processing via the $P V M$ module, plus passing of data back and forth to $D S 9$ via the XPA module, plus .... Oh, hell, if you're still using XSPEC instead, you deserve what you get. For an introduction, see http://space.mit.edu/home/mnowak/isis_vs_xspec/index.html.
} 

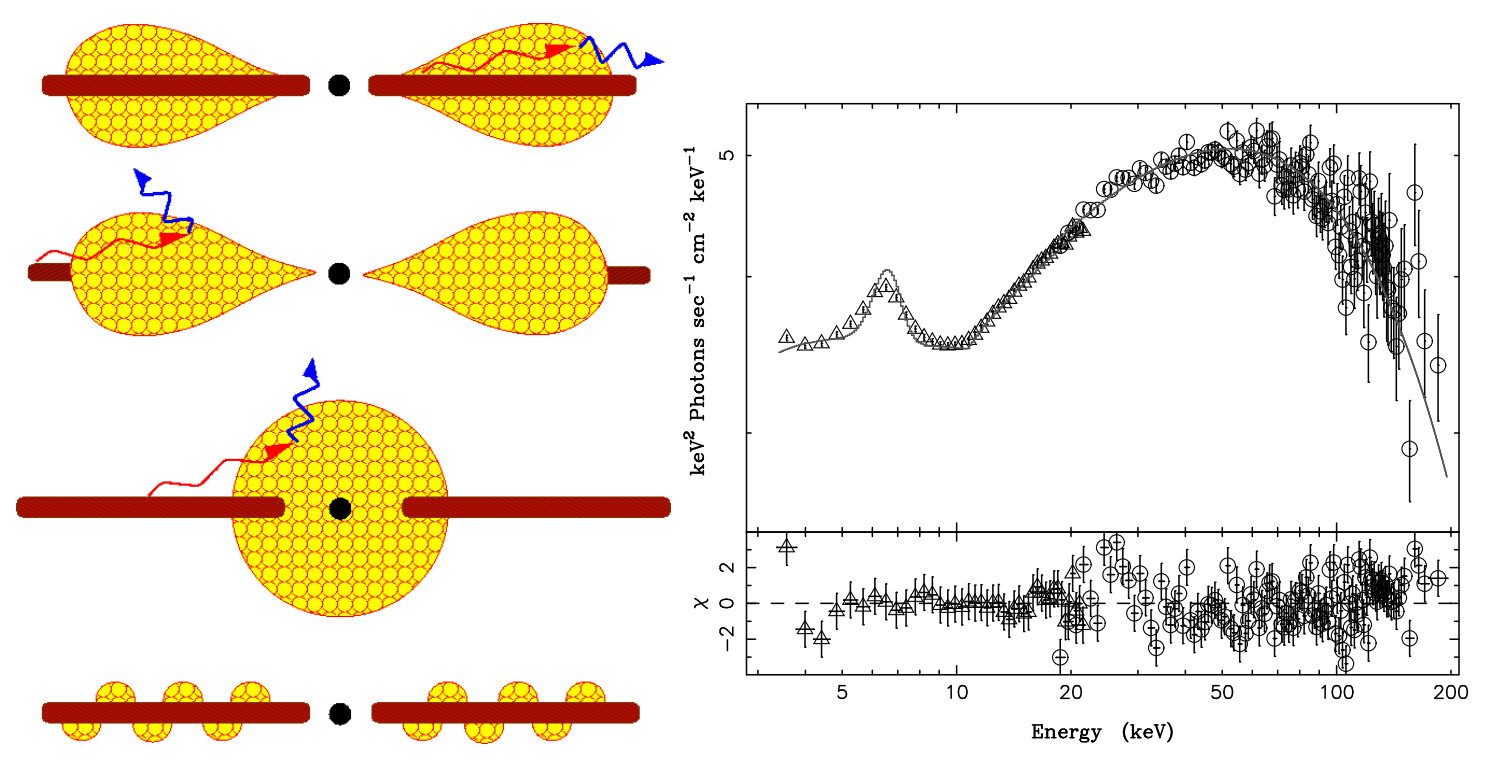

Figure 1: Left: A number of suggested coronal geometries. The middle two are currently very popular, e.g., in ADAF models; however, their revival in Compton codes was somewhat independent of ADAFs (see text). Right: 'Flux-corrected' (i.e., not following XSPEC's unfolding scheme - see text and Nowak et al. 2005) RXTE spectra of a very hard state of Cyg X-1.

A typical Cyg X-1 hard state spectrum, as observed by RXTE in the 3-200 keV band, is shown in Fig. 1. The salient features - present in all RXTE spectra of Cyg X-1 - are a low energy (3$10 \mathrm{keV}$ ) power law that hardens above $10 \mathrm{keV}$, a (typically) broad line feature near $6.4 \mathrm{keV}$, and an exponential rollover at high energies (usually $>20 \mathrm{keV}$ ). Cyg X-1 "soft states" also often require an additional soft component (which can be modeled with a simple phenomenological disk component). In fact, the simple phenomenological model as described above fits RXTE data better than any sophisticated Comptonization or jet model that we have tried [31]. As a corollary, including parameterizing absorption and a relative PCA/HEXTE normalization constant, any model that uses more than 11 free parameters to characterize the $R X T E$ data is likely over-parameterized.

Comptonization of soft (seed) disk photons in a hot $(\approx 100 \mathrm{keV})$ corona with $\tau_{e s}$ of order a few has long been proposed as a physical description of BHC spectra [7, 28]. Debate centers around the geometry, with 'sphere+disk' models currently being very popular (see Fig. 1). The 'revival' of the 'sphere+disk' geometry for models of Cyg X-1 in fact occurred independently of Advection Dominated Accretion Flow (ADAF) models, which also posit this geometry. It had been noted that unless the corona is 'photon starved' (i.e., only a fraction of seed photons enter the corona), it is simply too difficult to achieve temperatures high enough to produce spectra as hard as those seen in Cyg X-1 [5, 25]. 'Pill box' geometries (i.e., the bottom of Fig. 1), although photon starved, produce too much reflection, unless some process like relativistic beaming [1] is also invoked.

In [31], we successfully fit the RXTE Cyg X-1 spectra with the eqpair model of Coppi [2], if we include additional disk and line components (smeared reflection is included in the eqpair model). Fits to the hardest and softest spectra are shown in Fig. 2. The relevant coronal fit parameters then become the relative compactness (i.e., energy divided by radius) of the corona to 

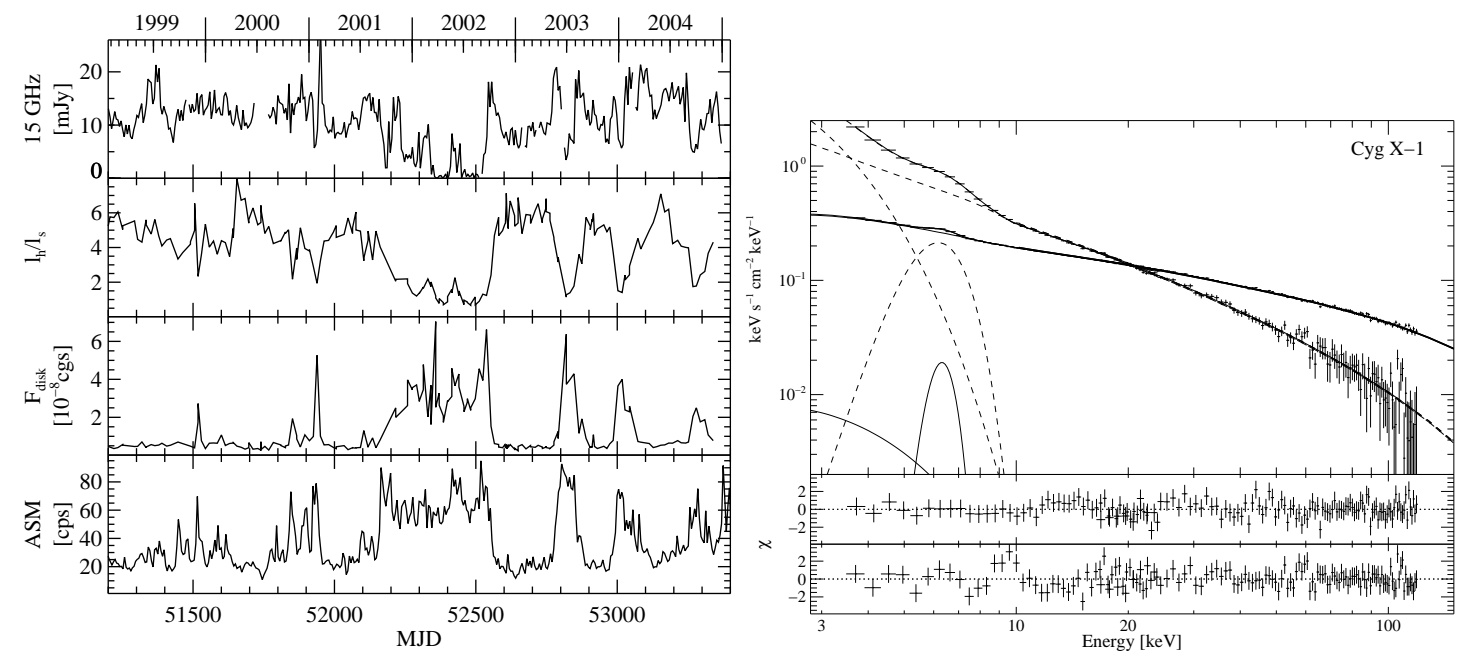

Figure 2: Left: ASM flux, radio flux, coronal compactness and disk flux (the latter two from phabs* (diskpn+eqpair+gauss) fits) from our pointed RXTE observations of Cyg X-1. Right: Our softest and hardest Cyg X-1 observations. (Sadly, the latter shown as the potentially misleading XSPEC unfolded spectra. The individual diskpn, eqpair, and gauss model components are also shown.)
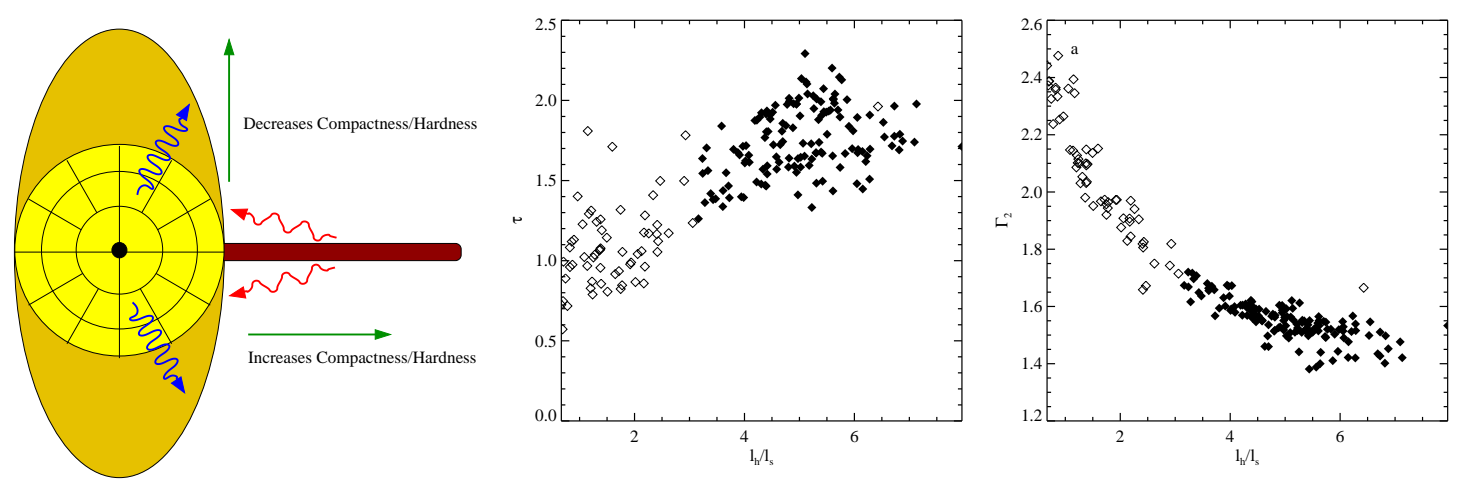

Figure 3: Left: Schematic of the sphere+disk coronal geometry, with expectations for how the compactness/hardness could change with simple changes of the geometric parameters. Middle: coronal optical depth vs. compactness ratio for our pointed observations of Cyg X-1. Here, and throughout, clear points will refer to the Remillard \& McClintock [26] power law photon index-based definition of the soft state $\left(\Gamma_{1}>2.2\right)$, while the solid points refer to the photon index-based definition of the hard state. Right: higher energy power law photon index $\left(\Gamma_{2}\right)$ vs. ratio of coronal compactness.

that of the disk, and the coronal optical depth. Other Comptonization models also work (with, for example, Compton $y$ parameter taking the role of relative compactness). Overall trends remain unchanged among these models, especially on "broad" features such as fluxes; however, absolute numbers (e.g., reflection fraction, Fe line strength and width) are altered, often in systematic ways [31]. Trends from the eqpair fits are shown in Fig. \&

Using the eqpair model in this way only roughly approximates the sphere+disk geometry, but it does provide good fits to the data. There is also a nearly one-to-one correspondence with 

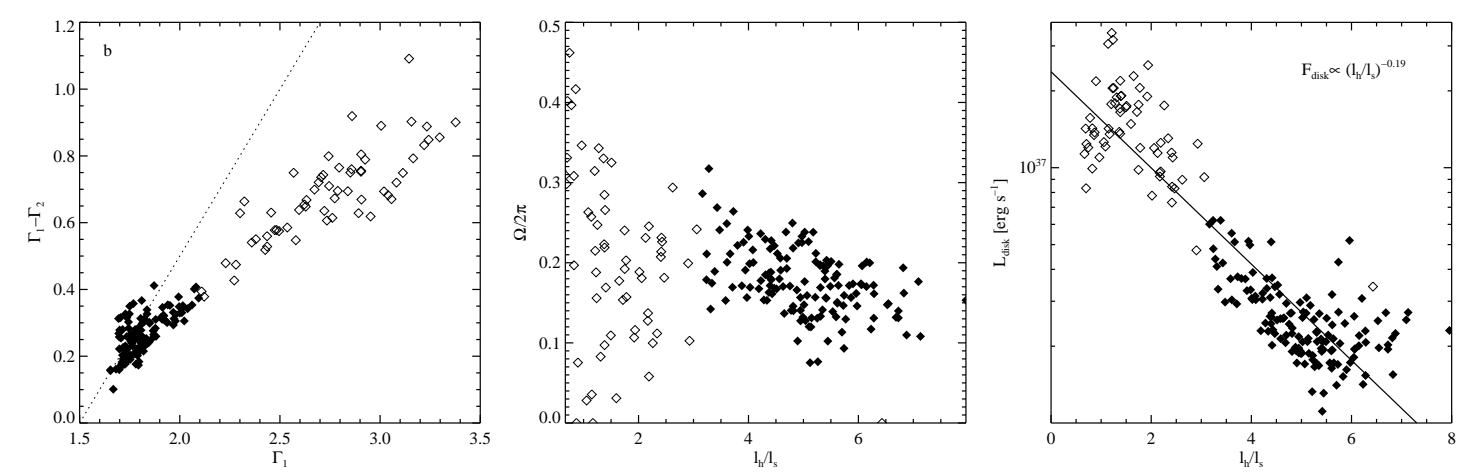

Figure 4: Left: Difference in photon indices vs. lower energy photon index for broken power law fits to Cyg X-1. Middle: Reflection fraction vs. compactness ratio for coronal model fits. Right: Disk flux vs. compactness ratio for coronal model fits.

broken power law fits (see Fig. 3). An important point to note here is that what is a very clear and distinct power law break vs. power law index correlation (Fig. (4) is not solely attributable to a reflection fraction-hardness correlation (i.e., [32]) in Comptonization models. Although a weak relation between reflection vs. coronal compactness (i.e., hardness) is seen, the $\Delta \Gamma-\Gamma_{1}$ correlation is more dominated by a disk flux-coronal compactness correlation (Fig. đ). That is, the break at $10 \mathrm{keV}$ is largely driven by the relation between two broad band continuum components being observed within the RXTE band. To be clear, the presence of the Fe line indicates that there must be reflection, but its exact value and its correlations cannot be determined independently from the broad-band continuum components assumed and fit in the 3-200 keV regime.

Within the context of the sphere+disk Comptonization model, aside from changing the energetics or optical depth of the corona, one can alter the spectral hardness by changing the size of the corona and/or the radius of the transition region between disk and corona (see Fig. 1). Such geometry changes have been inferred from spectral fits, e.g., the reflection-hardness correlations, but as we show above, that correlation is weak, is not truly self-consistently calculated within the context of the fit model, and it does depend upon the presumed Comptonization and reflection model (i.e., reflect ${ }^{\star}$ comptt models show a much more pronounced correlation, which is likely systematic rather than physical in nature; [31]). Thus, researchers have turned to timing features to search for further clues as to geometry changes in the Cyg X-1 system.

As we (and others) have shown, the Power Spectral Densities (PSD) of the X-ray variability of Cyg X-1 can be well-described as the sum of multiple, broad Lorentzian components [17], with four components dominating the fits (Fig. 6). The peak frequencies of these features are well-correlated with spectral hardness, with harder spectra corresponding to lower frequencies [24]. This is what one expects in sphere+disk models if the frequencies are indicative of characteristic disk time scales at the radius of the transition region between corona and disk, and this transition radius moves outward as the source becomes fainter and harder. If this is the case, then the highest frequency component at $\approx 40 \mathrm{~Hz}$ indicates that the transition region never moves beyond $\approx 40 \mathrm{GM} / \mathrm{c}^{2}$. (This is in contrast to many of the larger values found referenced for ADAF models. As such, these models have greatly scaled down their hypothesized coronal region sizes in recent years.) 

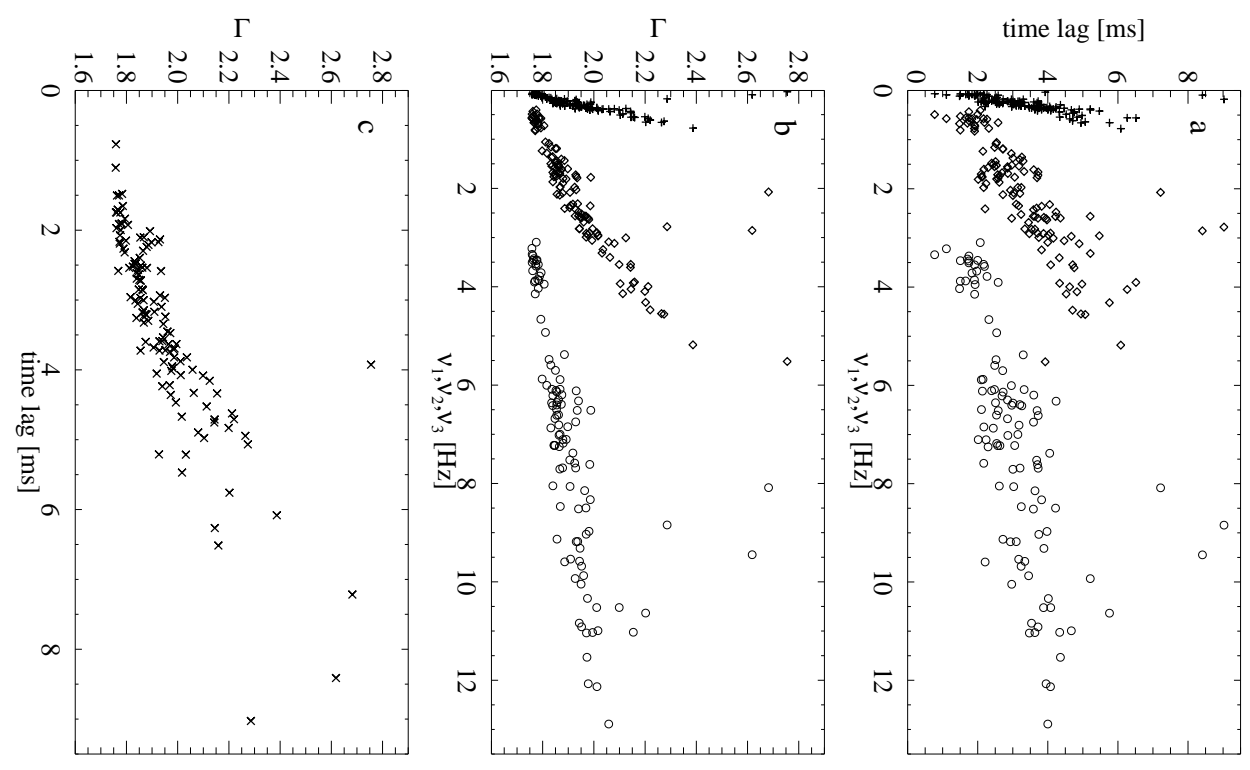

Figure 5: The dependence of characteristic power spectral density (PSD) frequencies upon photon index (from Pottschmidt et al. 2002; $\Gamma \approx \Gamma_{1}$ ). Also shown are time lags between hard and soft variability vs. both photon index and characteristic PSD frequency.
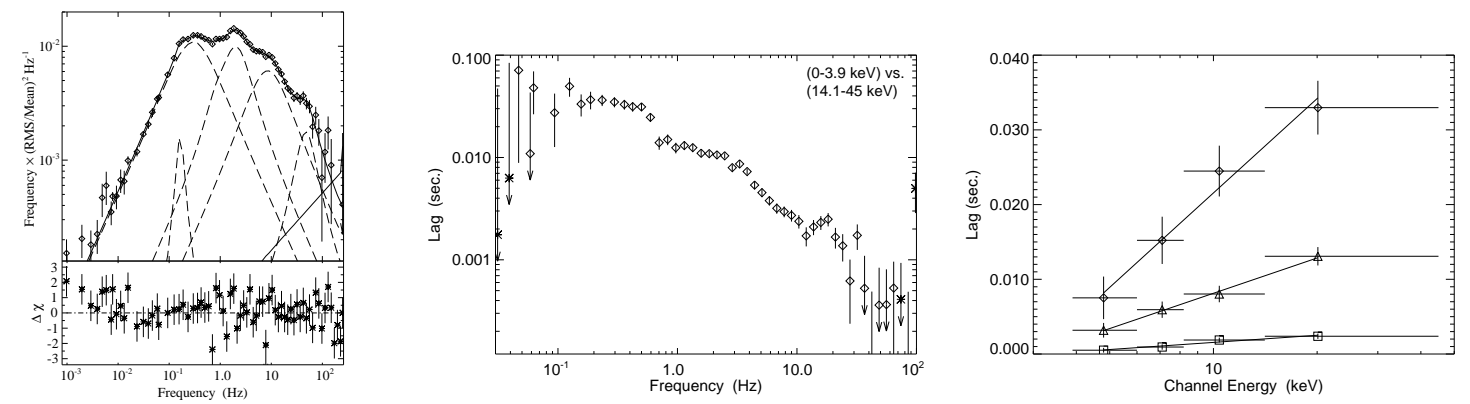

Figure 6: Left: PSD of Cyg X-1 fit with five Lorentzian features. The four most prominent ones seem to be persistent in the hard state PSD, and vary with spectral properties (see previous figure). Middle: Time lag between hard and soft X-ray variability for a hard state observation of Cyg X-1 (see Nowak et al. 1999). Note the peaks near the PSD peak frequencies. Right: The energy dependence of the hard X-ray variability lag for three different PSD frequencies (see Nowak et al. 1999).

Often neglected is the fact that while the characteristic variability time scales increase with hardness, the characteristic time lags between soft and hard X-ray variability decrease [24]. This has no obvious explanation in the scenario where the transition radius grows as the source fades into quiescence. Furthermore, the time lag between soft and hard X-ray variability seems to be composed of multiple components at different frequencies, very possibly associated with the individual Lorentzian components in the PSD (Fig. 6, [18, 17]). The time lag we observe may be in reality a composite of time lags and leads from independent components, and this possibility is absent in most models of these data. Additionally, it is known that the time lag has a logarithmic 

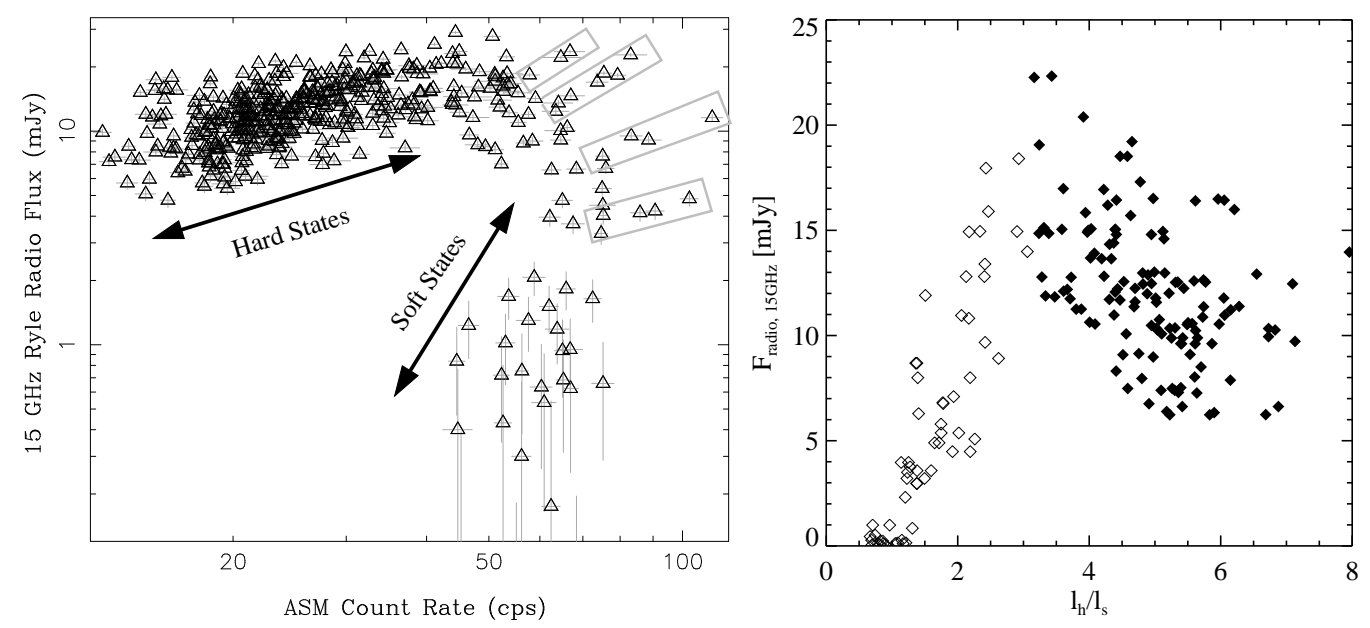

Figure 7: Left: $15 \mathrm{GHz}$ radio flux, measured with the Ryle radio telescope, vs. daily average ASM count rate for Cyg X-1(see Nowak et al. 2005). Boxes correspond to "failed state transitions". Right: Ryle radio flux vs. coronal compactness for our pointed observations of Cyg X-1.

dependence upon energy, and in the past this had been used to argue for Comptonization models (where logarithmic energy dependences are naturally expected); however, given the extremely long time scale of the lags relative to dynamical time scales, the inferred coronal size is unreasonably large [23]. The large magnitude of the time lag must be incorporated in any model.

Finally, in all of the above we have not addressed any of the radio data. Nearly all of our Cyg X-1 observations have simultaneous $15 \mathrm{GHz}$ radio data obtained with the Ryle radio telescope. As shown in Fig. 7, although the radio can fade with increasing flux/decreasing hardness, it rarely fully disappears in Cyg X-1. This is partly what we mean by saying that state transitions are not distinct in Cyg X-1 - there appears to be a continuum of observed properties between the hardest and softest spectra. In fact, there are only two properties where the Cyg X-1 state transitions do seem sharply defined. First, variability time lags seems to greatly lengthen in state transitions and failed state transitions [24]. Second, as shown in Fig. 7] (see also [31]), the slope of the radio/X-ray hardness correlation changes from one state to the other (although this figure, too, straightens out if one plots radio vs. hard X-ray flux; [22] ). In nearly all Compton models to date, the radio data, which clearly is an important aspect of the source properties, is an ad hoc add-on, with only vague arguments as to its correlation with the X-ray.

The need to self-consistently fit the radio and X-ray data together has given rise to jet models of BHC [16]. The earliest versions of these models were dominated solely by synchrotron radiation in the X-ray regime; however, for over three years now, these models have included synchrotron, synchrotron self-Compton (SSC), disk photons, and Comptonization of disk photons. Other reviews will go into these models in more detail; however, we will point out two salient facts. First, as shown in Fig. 8, these models not only fit the radio data, they also simultaneously fit the RXTE $\mathrm{X}$-ray data equally well as the Comptonization models. Second, like the Comptonization models, the X-ray is dominated by two broad-band continuum components that in part lead to the $10 \mathrm{keV}$ power law break. As opposed to being disk plus Comptonization components, these two compo- 


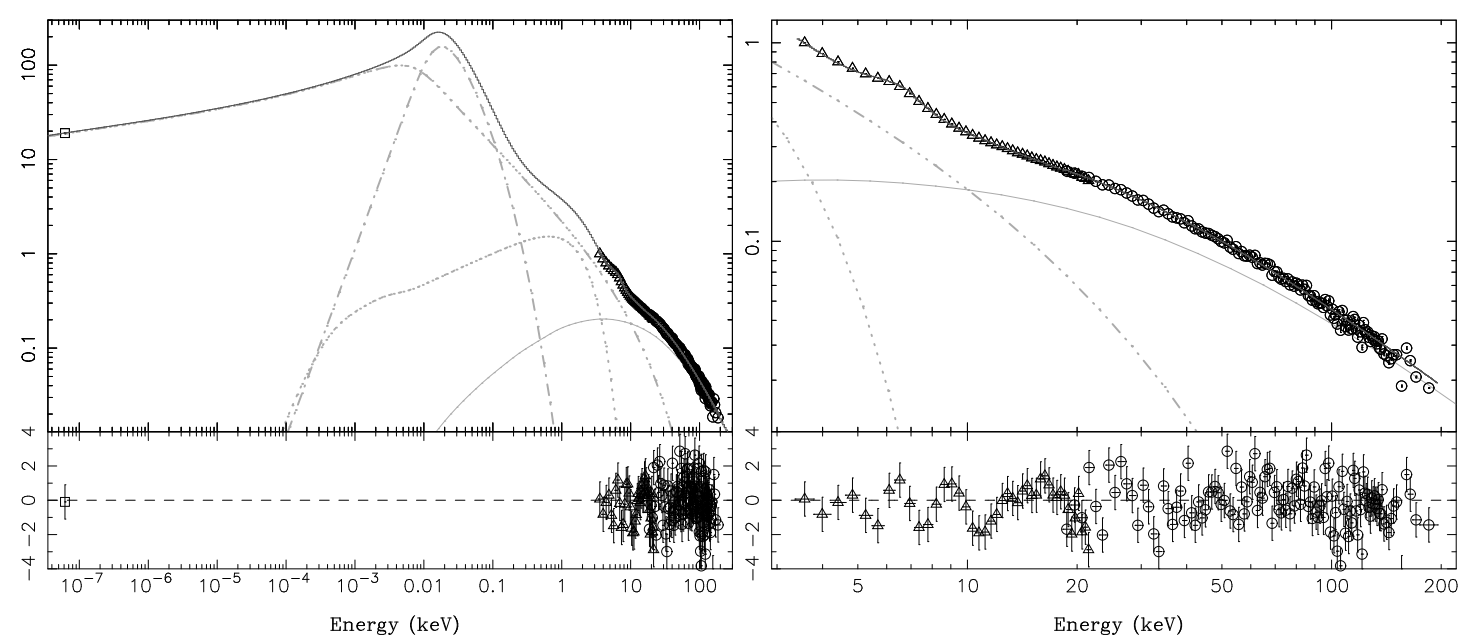

Figure 8: Jet model fit to radio and X-ray hard state data of Cyg X-1 (see Markoff, Nowak, \& Wilms 2005). The model components include disk emission, synchrotron, synchrotron self-Compton, and Comptonization of disk photons. In the X-ray regime, the models work equally as well as traditional corona models.
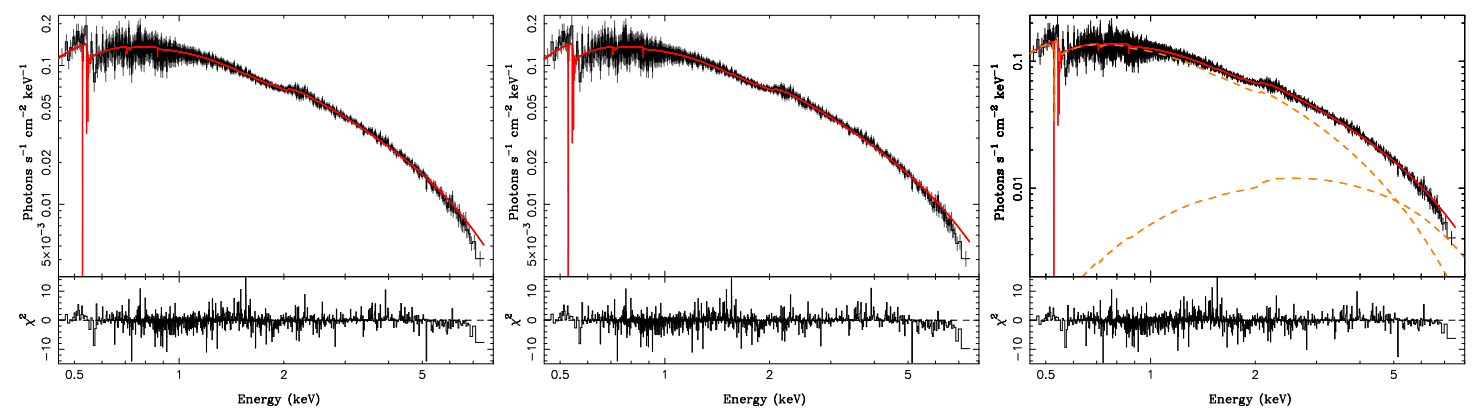

Figure 9: Left: The kerrbb model fit to the combined $M E G$ data of $4 U$ 1957+11. System distance, inclination, and mass were fixed (see text), but spectral hardening factor and black hole spin were left free. Middle: same data and model as on the left, except that now the black hole spin is fixed to $a^{*}=0$. Right: A disk plus Comptonization model (see text) fit to the combined MEG data. Dashed lines show the individual model components.

nents instead are synchrotron and SSC. (The upper break is also partly influenced by a Comptonized disk component). Again, as there is a broad Fe line, reflection must be a part of these spectra. The specific reflection and line parameters one fits, however, will change depending upon whether one assumes a Compton corona or X-ray emitting jet model.

\section{3. $4 \mathrm{U} 1957+11$}

Contrary to Cyg X-1, 4U $1957+11$ is a source that seems to spend nearly all of its time in a spectrally soft (i.e., "high") state. It shows evidence of a hard tail only at its highest luminosities [29], thus 4U 1957+11 cannot be used as a test of jet models. Instead, 4U 1957+11 provides us with an opportunity to test recent sophisticated disk atmosphere models that incorporate the effects 
of black hole spin on the spectra (e.g., [14]). If such models truly can unambiguously measure black hole spin as has been hypothesized [27], then they can be applied to systems that transit between hard and soft states to determine whether rapid spin is indeed a necessary component of jet production (thus testing the Fender Conjecture). We have obtained a simultaneous Chandra/RXTE observation, and furthermore have analyzed all RXTE observations from the archive. Below we apply the kerrbb disk model [14], which includes spin, to these spectra.

The Chandra spectra are shown in Fig. 9. Owing to the high resolution and the $0.4 \mathrm{keV}$ lower bound of our Chandra gratings observation, we can accurately measure the neutral column $\left(10^{21} \mathrm{~cm}^{-2}\right)$ in front of the source, which removes one potential source of ambiguity in the models. Although predominantly being merely phenomenological, the two-parameter diskbb model fits the data very well. Thus, similar to our point about broken power law fits to Cyg X-1, any model that attempts to describe the data with more than two parameters is likely over-determined. The kerrbb model has seven: system mass $(M)$, accretion rate $(\dot{M})$, distance $(D)$, inclination $(\theta)$, spectral hardening factor $\left(\mathrm{f}_{\mathrm{col}}\right)$, torque parameter $(\eta)$, and dimensionless black hole spin $\left(a^{*}\right)$.

In practical application of the kerrbb model, it is hoped that $M, D$, and $\theta$ can be determined via other observations and that $\mathrm{f}_{\mathrm{col}}$ and $\eta$ can be determined from theoretical considerations, which would leave only $\dot{M}$ and $a^{*}$ as fit parameters. The complex optical lightcurve of $4 \mathrm{U} 1957+11$ implies an inclination of $\sim 75^{\circ}$ [11]; however, the system mass and distance are completely unknown. The lowest luminosity RXTE observations do not transit to the hard state; although, an upturn in the fitted disk normalization from diskbb+powerlaw models (Fig. 10) indicates they may be very near the transition, expected to be at $\approx 3 \% L_{\text {Edd }}$ [15]. Thus the least massive/closest $4 \mathrm{U} 1957+11$ could be is $3 \mathrm{M}_{\odot}$ at $10 \mathrm{kpc}$ (used in all figures shown here). The expected distance then scales as the square root of the mass (e.g., $16 \mathrm{M}_{\odot}$ at $23 \mathrm{kpc}$ ).

Simple diskbb fits to the spectra yield very high temperatures $(k T \sim 1.7 \mathrm{keV})$, and low normalizations $(\sim 8)$. If one fixes $f_{\text {col }}$ in the kerrbb model, these diskbb parameters are only achievable with a combination of large distance, high accretion rate, and high spin. (High spin becomes more crucially needed if one increases the mass of and distance to $4 \mathrm{U} 1957+11$.) A high spin model (with $\mathrm{f}_{\mathrm{col}} \sim 1.1$ ) is shown in Fig. 9. Note, however, that we can find a nearly equally good fit with $a^{*}=0$ if we allow $\mathrm{f}_{\mathrm{col}}=3.3$ (Fig. 9). Thus, at a minimum, one must be absolutely convinced that there is a strong theoretical motivation for specific values of $\mathrm{f}_{\mathrm{col}}$ and $\eta$ if disk models are to be used to 'measure' spin.

As opposed to the Chandra data, where $a^{*} \approx 1$ and $a^{*}=0$ are virtually indistinguishable, the very high statistics $R X T E$ data seem to require $a^{*} \approx 1$ (and find $\mathrm{f}_{\text {col }} \approx 1.1$ ). If one sets the color correction factor to the 'theoretically preferred' value of $\mathrm{f}_{\text {col }}=1.7$, and requires that the faintest observations have a luminosity of $\approx 3 \% L_{\mathrm{Edd}}$, then the $R X T E$ data require $a^{*} \approx 1, D \approx 23 \mathrm{kpc}$, $M \approx 16 M_{\odot}$. (The degeneracy among all these models is partly indicative of the fact that the produced spectra are rather smooth, with no specific sharp features unique to $a^{*}=1$, for example.) If such models can usefully be used to measure $a^{*}$, then a prediction of the kerrbb model, based on the RXTE data, is that future observations should find $4 \mathrm{U} 1957+11$ to be approximately $16 \mathrm{M}_{\odot}$ and near $23 \mathrm{kpc}$, i.e., well into the galactic halo.

A further serious issue in applying the kerrbb model arises in looking at both the Chandra and RXTE data. If one allows a hardening due to Comptonization (here, modeled as the addition of the comptt model with a fixed coronal temperature and seed photon temperature frozen to the 

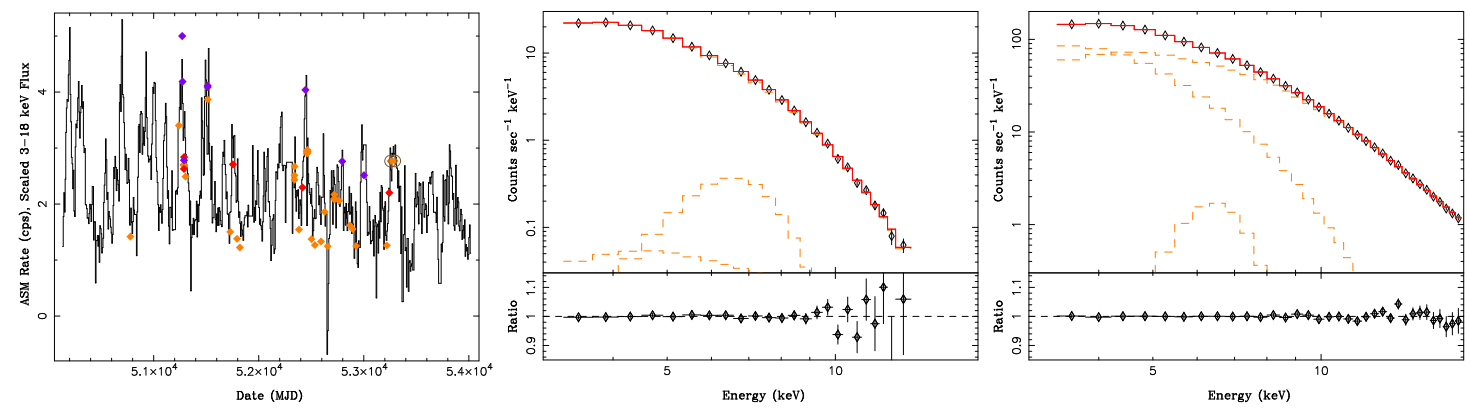

Figure 10: Left: All Sky Monitor lightcurve for 4U 1957+11 with scaled 3-18 keV PCA flux from pointed observations overlaid. RXTE observations simultaneous with Chandra and XMM-Newton are circled. Middle, Right: Count rate spectra for the softest/faintest (middle) and hardest/brightest (right) of the RXTE observations, fit with the kerrbb+comptt+gaussian model. Dashed lines show the individual model components folded through the detector response.
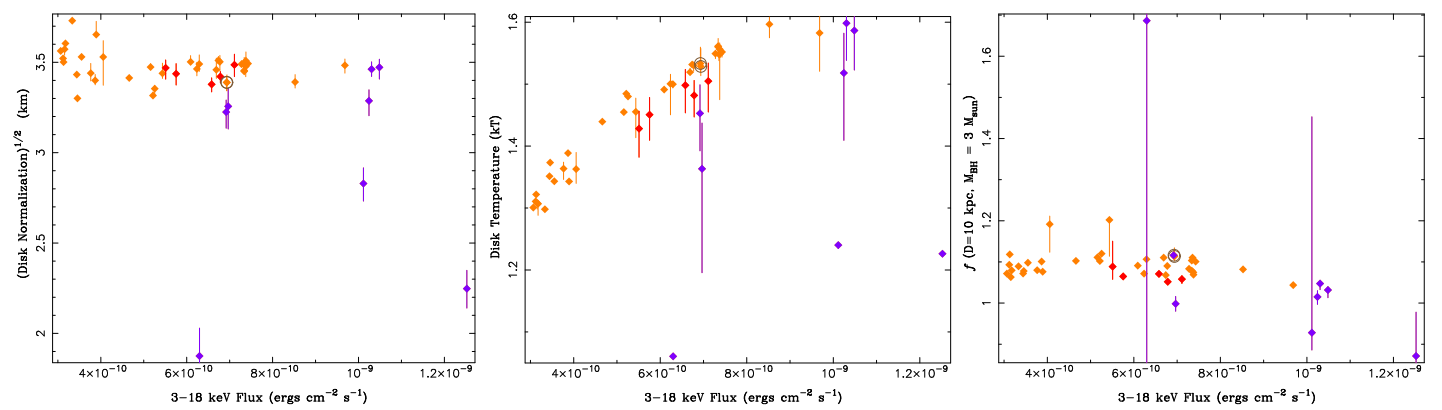

Figure 11: Left: Square root of disk normalization vs. PCA flux for diskbb+powerlaw fits to RXTE observations of $4 \mathrm{U} 1957+11$. Middle: disk temperature vs. PCA flux for diskbb+comptt fits to 4U 1957+11. Right: Spectral hardening factor vs. PCA flux for kerrbb+comptt fits to 4U 1957+11. (For the latter, we assume a black hole mass of $3 M_{\odot}$, a distance of $10 \mathrm{kpc}$, and an inclination of $75^{\circ}$.)

best-fit diskbb temperature), one achieves an even better fit to the Chandra data than either the diskbb or kerrbb models alone. Furthermore, the best fit diskbb temperature decreases to $1.3 \mathrm{keV}$, and the best fit normalization increases to 15 . That is, the very parameters driving the need for high spin are fundamentally altered to values indicative of much lower spin. The fit to the Chandra data essentially leaves the spin parameter unconstrained in a systematic, rather than a statistical, sense.

RXTE spectra, however, still require a high spin parameter. As shown in Fig. 11, there are periods when the disk temperature and normalization drop dramatically, and the spectrum becomes dominated by the Compton component (Fig. 10). These correspond to $4 \mathrm{U} 1957+11$ entering the "very high" or "steep power law" state. Otherwise, flux variations are predominantly driven by disk temperature changes in diskbb models, or accretion rate changes in kerrbb models. Note in Figs. 9 and 10 that even when dominated by the Compton component, the $4 \mathrm{U} 1957+11$ spectrum is very soft, and only differentiated from the disk spectrum by a decrease in spectral curvature ${ }^{2}$.

\footnotetext{
${ }^{2}$ Note that we also include a weak Fe line, likely due to galactic emission, in the fits. When the kerrbb model has
} 

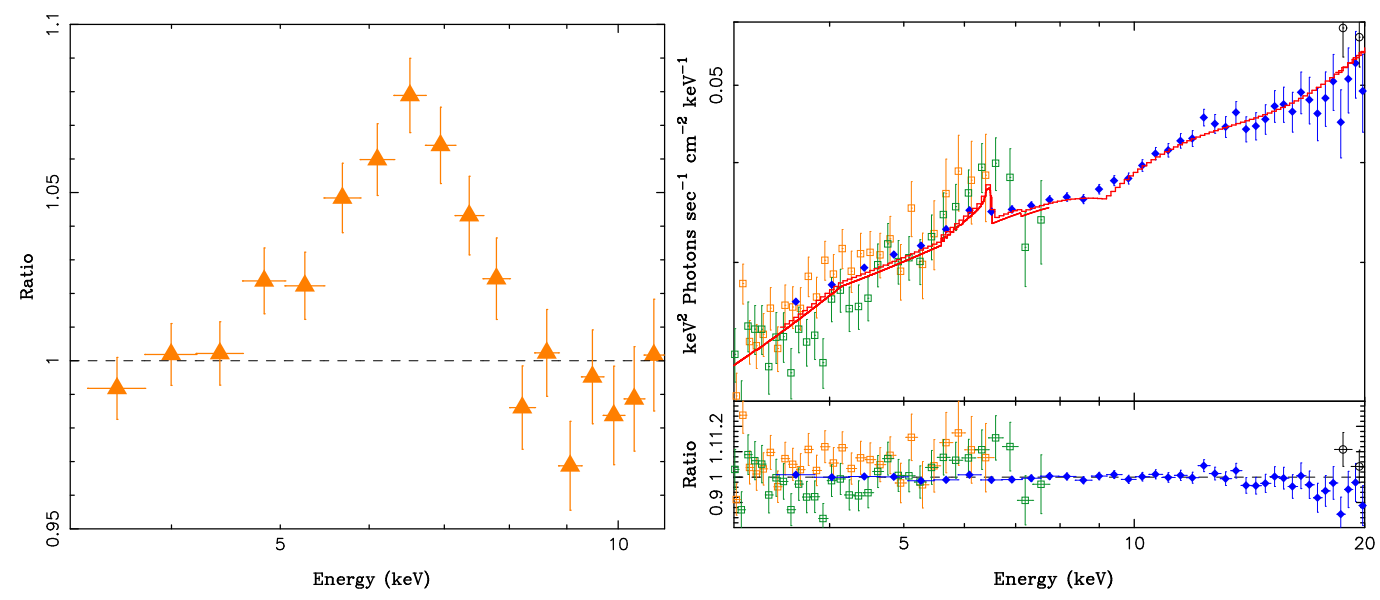

Figure 12: Left: Fe line residuals from an RXTE observation of a GX339-4 hard state as it enters quiescence. The line remains broad, contrary to the expectation of ADAF models, despite the low flux (see Nowak, Wilms, \& Dove 2002). Right: An even fainter hard state RXTE/Chandra observation of GX339-4, potentially showing a broad line (Nowak et al., in prep.)

In Fig. 11 we also see that as the flux increases, the fitted spectral hardening factor, $\mathrm{f}_{\mathrm{col}}$, decreases. That is, Comptonization is acting to harden the disk spectrum, rather than it being a necessary parameter in the disk atmosphere model itself. As the flux decreases, the hardening factor, $f_{c o l}$, smoothly increases, perhaps asymptoting to a 'pure disk' value. Given the fact even the Compton-dominated spectra are so soft, and there seems to be a continuous evolution of $\mathrm{f}_{\text {col }}$, can one really be sure that the lowest flux spectra are indeed "pure" disk spectra? How does one know that there isn't a "residual corona" that merely mimics the effects of high spin in the disk model? For these reasons, I am very skeptical that such models will ever usefully "measure" spin. (However, if independent observation determines that $4 \mathrm{U} 1957+11$ is indeed a $16 \mathrm{M}_{\odot}$ black hole at $23 \mathrm{kpc}$, I may become a believer ...)

\section{GX339-4}

GX339-4 has been a very important source in establishing the relationship between hard Xray flux and steady radio jet activity [12, 8, 3, §]. We have extensively studied the spectra and variability properties of this source ourselves, and have successfully fit both Comptonization and jet (and, of course, broken power law) models to the hard state spectra [30, 19, 17, 21, 22, 16]. Here, I will only briefly touch upon two faint, hard state observations of GX339-4.

The 'sphere+disk' Comptonization models posit a transition radius between inner corona and outer disk, and likewise ADAF models similarly posit a transition radius from outer, efficient flow to inner, inefficient flow. The former models do not set a specific radius for this transition (although as discussed earlier, timing data of Cyg X-1 indicate that it cannot occur at $R>40 G M / c^{2}$ ). The ADAF models have traditionally posited greater transition radii, with the radius possibly increasing

been fit to other sources, researchers have often required stronger lines, smeared edges, and even more prominent power law components than required here, adding greatly to systematic uncertainties in the use of these disk models [27]. 

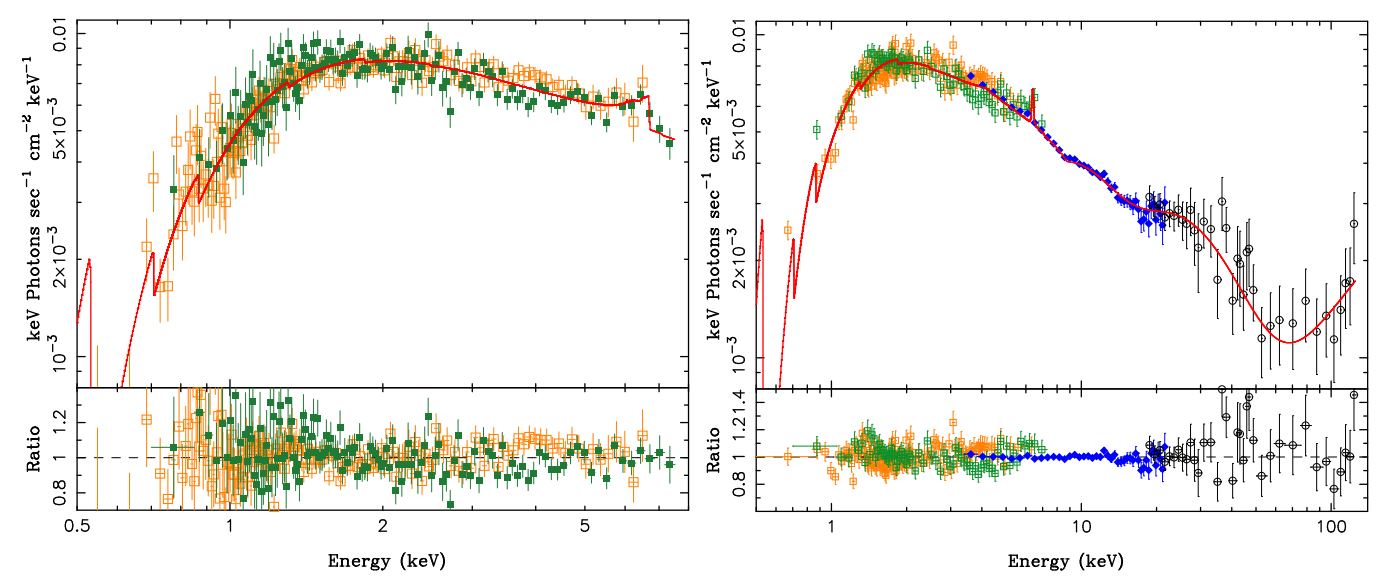

Figure 13: Left: The broad-band Chandra spectra. The model is consistent with a disk whose inner edge extends to the marginally stable orbit, plus a broad line. Right: The broad-band Chandra/RXTE spectra. The observed (multiple humped) curvature, and flat high energy tail, are real. This is not your grandmother's broken power law, and does not fit either a simple corona or jet model (Nowak et al. in prep.)

as the source falls further into quiescence. Thus, especially for the latter models, we do not expect to see broad Fe lines persist into quiescence. GX339-4 shows at least one, possibly two, counterexamples to that ADAF expectation.

As discussed in [21], and shown in Fig. 12, the 1999 fade into quiescence saw the Fe line remain strong and persistently broad. Despite the fact that the source was approximately a factor of 10 lower in flux from its hard state transition level, the best fits indicated that the inner edge of the line emission region was consistent with the marginally stable orbit. This is completely inconsistent with ADAF theory.

More recently, during the 2005 fade into quiescence, we obtained a simultaneous Chandra/RXTE observation at an even fainter hard state flux level. As shown in Fig. 12, these data are also consistent with a relativistically broadened line, counter to the expectations of ADAF models (Nowak et al., in prep.). Here, however, owing to the faint nature of these spectra, the fits are more uncertain (galactic ridge emission must be carefully subtracted from the RXTE spectra); however, the broad line does seem to be preferred in the Chandra data. Likewise, if one fits a diskbb+powerlaw model to solely the Chandra data, the fitted disk also prefers an inner radius consistent with the marginally stable orbit, rather than the much larger disk radii often fit in hard state sources (Fig. 13).

The 1999 fade into quiescence was 'regular' in all respects, and was instrumental in defining the the $F_{\text {radio }} \propto F_{X-\text { ray }}^{0.7}$ correlation between radio and X-ray fluxes in hard state BHC [ $\llbracket$ ]. The presence of the broad line in those data is also unambiguous, and thus is challenging for ADAF theory. On the other hand, the 2005 fade into quiescence was very unusual. As discussed by S. Corbel in these proceedings, a few weeks before the Chandra/RXTE observation, GX339-4 'fell off' the usual radio/X-ray correlation, with the radio decreasing much more rapidly. Relative to the X-ray flux (which itself was very low, given that the observations occurred only three weeks after the hard state transition), the radio was approximately a factor of ten too faint for the usual 
correlation.

Furthermore, the broad-band X-ray spectra themselves, as shown in Fig. 13, are highly unusual. The data show a great deal of curvature and spectral breaks, and there is a very spectrally flat high energy tail. All of these features are real, and are not artifacts of the unfolding process. (Remember, we're not using XSPEC ...). We have also been very careful in the background subtraction of the galactic ridge emission. These data simply represent a very unusual, radio-weak hard state spectrum. Currently, we have no good model with either typical corona or typical jet models. (Broken power law models don't even work well here.) Why GX339-4 failed to act as a 'proper' microquasar in this particular outburst decay remains a mystery.

\section{Acknowledgments}

I'd like to thank my coauthors and collaborators on the above cited projects: Stephane Corbel, James Dove, Thomas Gleissner, Adrienne Juett, Sera Markoff, Guy Pooley, Katja Pottschmidt, \& Jörn Wilms. Intelligent insight is largely thanks to them, ranting is wholly my own. If there were less of the latter, and this proceedings weren't already a month late, many of them would be coauthors here. I would like to thank the organizers for inviting me, and NASA Grant SV3-73016 for funding this work.

\section{References}

[1] Andrei M. Beloborodov, Plasma ejection from magnetic flares and the X-ray spectrum of Cygnus X-1, ApJ 510, L123-L126 (1999).

[2] Paolo Coppi, PASP Conference Series, 161, 375, 1999.

[3] S. Corbel, R. P. Fender, A. K. Tzioumis, M. Nowak, V. McIntyre, P. Durouchoux, and R. Sood, Coupling of the $X$-ray and radio emission in the black hole candidate and compact jet source GX 339-4, A\&A 359, 251-268 (2000).

[4] S. Corbel, M. A. Nowak, R. P. Fender, A. K. Tzioumis, and S. Markoff, Radio/X-ray correlation in the low/hard state of GX 339-4, A\&A 400, 1007-1012 (2003).

[5] James B. Dove, Jörn Wilms, Michael G. Maisack, and Mitchell Craig Begelman, Self-consistent thermal accretion disk corona models for compact objects: II. Application to Cygnus X-1, ApJ 487, 759-768 (1997).

[6] James B. Dove, Jörn Wilms, Michael A. Nowak, Brian A. Vaughan, and Mitchell C. Begelman, RXTE observations of Cygnus X-1. I. spectral analysis, MNRAS 298, 729 (1998).

[7] Douglas M. Eardley, Alan P. Lightman, and Stuart L. Shapiro, Cygnus X-1: A two-temperature accretion disk model which explains the observed hard X-ray spectrum, ApJ 199, L153 (1975).

[8] R. Fender, S. Corbel, T. Tzioumis, V. McIntyre, D. Campbell-Wilson, M. Nowak, R. Sood, R. Hunstead, A. Harmon, P. Durouchoux, and W. Heindl, Quenching of the radio jet during the X-ray high state of GX 339-4, ApJ 519, L165-L168 (1999).

[9] T. Gleissner, J. Wilms, G. G. Pooley, M. A. Nowak, K. Pottschmidt, S. Markoff, S. Heinz, M. Klein-Wolt, R. P. Fender, and R. Staubert, Long term variability of Cyg X-1. III. radio-X-ray correlations, A\&A 425, 1061-1068 (2004). 
[10] T. Gleissner, J. Wilms, K. Pottschmidt, P. Uttley, M. A. Nowak, and R. Staubert, Long term variability of Cyg X-1. II. the rms-flux relation, A\&A 414, 1091-1104 (2004).

[11] P. J. Hakala, P. Muhli, and G. Dubus, Evidence for evolving accretion disk structure in 4U1957+115, MNRAS 306, 701 (1999).

[12] D. C. Hannikainen, R. W. Hunstead, D. Campbell-Wilson, and R. K. Sood, MOST radio monitoring of GX 339-4, A\&A 337, 460 (1998).

[13] J. C. Houck and L. A. Denicola, ISIS: An Interactive Spectral Interpretation System for high resolution X-ray spectroscopy in proceedings of ASP Conf. Ser. 216: Astronomical Data Analysis Software and Systems IX, , vol. 9, 2000, p. 591.

[14] L.-X. Li, E. R. Zimmerman, R. Narayan, and J. E. McClintock, Multitemperature blackbody spectrum of a thin accretion disk around a Kerr black hole: Model computations and comparison with observations, ApJS 157, 335-370 (2005).

[15] T. J. Maccarone, Do X-ray binary spectral state transition luminosities vary?, A\&A 409, 697-706 (2003).

[16] S. Markoff, M. Nowak, and J. Wilms, Going with the flow: Can the base of jets subsume the role of compact accretion disk coronae?, ApJ 635, 1203-1216 (2005).

[17] Michael A. Nowak, Are there three peaks in the power spectra of GX 339-4 and Cyg X-1?, MNRAS 318, 361-367 (2000).

[18] Michael A. Nowak, Brian A. Vaughan, Jörn Wilms, James Dove, and Mitchell C. Begelman, RXTE observations of Cygnus X-1: II. Timing analysis, ApJ 510, 874-891 (1999).

[19] Michael A. Nowak, Jörn Wilms, , and James B. Dove, Low luminosity states of the black hole candidate GX 339-4. II. Timing analysis, ApJ 517, 355-366 (1999).

[20] Michael A. Nowak and Jörn Wilms, On the enigmatic source V1408 Aql (=1957+11), ApJ 522, 476-486 (1999).

[21] Michael A. Nowak, Jörn Wilms, and James B. Dove, Coronal-temporal correlations in GX 339-4: Hysteresis, possible reflection changes, and implications for ADAFs, MNRAS 332, 856-878 (2002).

[22] Michael A. Nowak, Jörn Wilms, Sebastian Heinz, Guy Pooley, Katja Pottschmidt, and Stephane Corbel, Is the 'IR coincidence' just that?, ApJ 626, 1006-1014 (2005).

[23] Michael A. Nowak, Jörn Wilms, Brian A. Vaughan, James Dove, and Mitchell C. Begelman, RXTE observations of Cygnus X-1: III. Comparisons to Compton corona and ADAF models, ApJ 515, 726-737 (1999).

[24] K. Pottschmidt, J. Wilms, M. A. Nowak, G. G. Pooley, T. Gleissner, W. A. Heindl, D. M. Smith, R. Remillard, and R. Staubert, Long term variability of Cyg X-1 (1998 to 2001) I. systematic spectral-temporal correlations in the hard state, A\&A 407, 1039-1058 (2003).

[25] J. Poutanen, J. H. Krolik, and F. Ryde, The nature of spectral transitions in accreting black holes: The case of Cyg X-1, MNRAS 221, 21p (1997).

[26] R. A. Remillard and J. E. McClintock, X-ray properties of black-hole binaries, Annual Review of Astronomy and Astrophysics 44, 49-92 (2006).

[27] R. Shafee, J. E. McClintock, R. Narayan, S. W. Davis, L.-X. Li, and R. A. Remillard, Estimating the spin of stellar-mass black holes by spectral fitting of the X-ray continuum, ApJ 636, L113-L116 (2006). 
[28] R. A. Sunyaev and J. Trümper, Hard X-ray spectrum of Cyg X-1, Nature 279, 506 (1979).

[29] Rudy Wijnands, Jon Miller, and Michiel van der Klis, $4 U$ 1957+11: a persistent low-mass X-ray binary and black-hole candidate in the high state?, MNRAS 331, 60-70 (2002).

[30] J. Wilms, M. A. Nowak, J. B. Dove, R. P. Fender, and T. di Matteo, Low luminosity states of the black hole candidate GX 339-4. I. ASCA and simultaneous radio/RXTE observations, ApJ 522, 460-475 (1999).

[31] Jörn Wilms, Michael Nowak, Katja Pottschmidt, Guy G. Pooley, and Sonia Fritz, Long term variability of Cygnus X-1. IV. Spectral evolution 1999-2004, A\&A 447, 245-261 (2006).

[32] Andrzej A. Zdziarski, Piotr Lubiński, and David A. Smith, Correlation between Compton reflection and X-ray slope in Seyferts and X-ray binaries, MNRAS 303, L11-L15 (1999). 